\title{
Towards Building an Optimal Demand Response Framework for DC Distribution Networks
}

\author{
Hamed Mohsenian-Rad, Member, IEEE and Ali Davoudi, Member, IEEE
}

\begin{abstract}
Direct current (DC) power systems have recently been proposed as a promising technology for distribution networks and microgrids. By eliminating unnecessary conversion stages, DC distribution systems can enable seamless integration of natively DC devices such as photovoltaic cells and batteries. Moreover, DC technologies can overcome several disadvantages of alternating current (AC) distribution systems, such as synchronization requirements and reactive power compensation. Therefore, in this paper, the first steps are taken towards designing demand response programs for DC distribution networks. The idea is to adjust the internal parameters of power electronics loads to ensure reliable and efficient operation of the DC distribution system. In this regard, first, an optimization-based foundation is proposed for demand response in DC distribution networks in presence of distributed generators. Then, the formulated problem is solved using both centralized and decentralized approaches, where the latter requires devising a pricing mechanism. Finally, simulation results are presented to assess the performance and to gain insights into the proposed demand-response paradigm.
\end{abstract}

Keywords: DC distribution networks, power electronics load, demand response, distributed generation, convex optimization.

\section{INTRODUCTION}

Demand response (DR) programs are designed to control the consumer resources in response to changes in the grid's operating conditions [1], [2]. The majority of prior studies on demand response do not take into account the characteristics of the underlying physical power system. Instead, they mainly focus on balancing the load across time to reduce the load at peak hours in large networks, e.g., see [3]-[7]. However, there are also some recent studies that have incorporated the impact of grid topology, power flow equations, and voltage control into the design of DR programs to tackle the challenges at the distribution level. So far, the focus has been only on alternating current (AC) distribution networks, e.g., see [8], [9]. In contrast, in this paper, demand response programs for direct current (DC) distribution networks are investigated.

Traditionally, DC power systems have been used in telecommunications, naval ships, and industrial systems [10], [11]. More recently, they are proposed also for microgrids [12] and distribution networks [13]-[15]. DC distribution networks can offer several important advantages. For example, they can enable more efficient interconnection of most energy storage units and renewable energy sources, such as photovoltaic (PV) systems, batteries, and fuel cells, that are natively DC sources.

H. Mohsenian-Rad is with the Department of Electrical Engineering at the University of California, Riverside, CA, USA, e-mail: hamed@ee.ucr.edu. A Davoudi is with the Department of Electrical Engineering at the University of Texas, Arlington, TX, USA, e-mail: davoudi@uta.edu. H. Mohsenian-Rad is the corresponding author for this paper. This work was supported by the National Science Foundation (NSF) Grants 1253516, 1137354, and 1319798.
Moreover, many of the emerging new loads are electronic DC loads (e.g., in data centers). Even some of the traditionally AC loads, such as induction machines, can appear as DC devices when controlled by drive systems [16]. Hence, DC distribution systems improve conversion efficiency by eliminating the ACDC conversion stage. DC microgrids are also shown to have about two orders-of-magnitude more availability compared to their AC counterparts, thus making them ideal candidates for mission critical applications [17], [18]. Finally, using DC technologies in distribution networks can overcome several disadvantages of AC technologies, including synchronization requirements, reactive power flow, and harmonics [15].

Given the benefits of DC distribution networks and the important role of demand response in the future smart grid, in this paper, the first steps are taken towards designing demand response programs for DC distribution systems and DC microgrids. In this regard, the recent advancements in power electronics [19]-[21] are combined with techniques from convex optimization and dual decomposition [22] to build a new foundation for optimal demand response in DC distribution networks and DC microgrids. Both centralized and decentralized design approaches are investigated, where the latter requires devising a pricing mechanism. The main contributions in this paper can be summarized as follow:

- A New Demand Response Paradigm: While most prior studies on demand response either do not consider the characteristics of the underlying physical power system or they assume that the underlying power system is AC, in this paper, a new demand response paradigm is proposed that is designed specifically for DC distribution networks.

- Centralized Design: A new optimization-based framework is developed to select the internal parameters of power electronics loads to assure desirable operation of the DC distribution network. This design takes into account the power flow equations, power draw limits of DC sources, DC-DC conversion, solar panels, solar irradiance, and proportional fairness. The formulated optimization problem is shown to be convex and tractable.

- Decentralized Design and Pricing: An algorithm and a pricing method are developed to implement the proposed demand response design in a decentralized fashion.

- Computer Simulations: Various computer simulations are conducted to evaluate the designs and to gain insights.

Compared to the conference paper in [23], the following aspects are new in this journal version. First, a detailed model for solar panels is incorporated in the problem formulation. Second, the impact of permissible duty cycles of DC-DC converters is considered in order to make the new design 


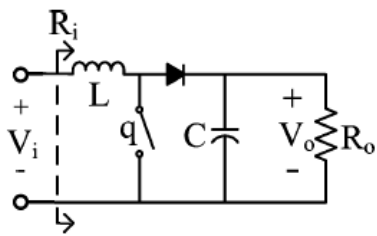

(a)

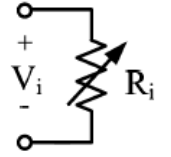

(b)
Fig. 1. A DC-DC boost converter can be seen as a power electronics load with variable resistor. The effective external resistor $R_{i}$ is controlled by setting the duty cycle for switch $q$ [21]. A buck converter can be modeled similarly.

more appropriate for a practical implementation. Third, new simulation results, in particular in Sections V-D and V-E, are added to further strengthen the analysis.

The rest of this paper is organized as follows. The fundamental system model is explained in Section II. The optimal demand response problem is formulated in Section III. Decentralized design is discussed in Section IV. Simulation results are given in Section V. The paper is concluded in Section VI.

\section{SYSTEM MODEL}

With the increasing penetration of power electronics loads, i.e., loads that are supplied with power electronics converters, the internal load characteristics can be controlled to reflect a desirable effective impedance [19], [20]. In particular, a power electronics load with a switch-mode converter can be seen by the rest of the grid as a variable resistor load, as shown in Fig. 1 [21]. For the boost converter example in this figure, the relationship between the internal resistance $R_{o}$ and the effective external resistance $R_{i}$ can be written as

$$
R_{i}=(1-D)^{2} R_{o}
$$

where $D \in[0,1)$ denotes the duty cycle of the active switch component $q$. A similar variable resistor load model can be found for pulse-width modulation (PWM) rectifiers that feed DC distribution systems from AC sources [24, Chapter 18]. By controlling the variable resistors for all power electronics loads, we can affect the power delivered to each user, the power drawn from each source, the power loss on each line, and the voltage level at each bus. Therefore, this system-level formulation can help define a demand-response paradigm.

\section{A. Formulation of a DC Power System}

Consider a DC power system such as the examples that are shown in Fig. 2. Let $\mathcal{N}$ denote the set of all DC buses. A DC power source at bus $k$ is modeled using its Thevenin's representation with a fixed voltage $V_{k}^{s}$, a fixed internal resistor $R_{k}^{s}$, and a maximum power limit $P_{k}^{\max }$. If there does not exist any source at bus $k$, then we simply assume that $R_{k}^{s}=\infty$. Next, let $\mathcal{L} \subset \mathcal{N}$ denote the set of load buses. Each load bus $i \in \mathcal{L}$ serves a power electronics load with an effective variable resistor $R_{i}$. Using the Kirchhoff's current law, we have

$$
\frac{V_{i}}{R_{i}}+\frac{V_{i}-V_{i}^{s}}{R_{i}^{s}}+\sum_{k \in \mathcal{N}_{i}} \frac{V_{i}-V_{k}}{R_{i k}}=0, \quad \forall i \in \mathcal{L},
$$

and

$$
\frac{V_{j}-V_{j}^{s}}{R_{j}^{s}}+\sum_{k \in \mathcal{N}_{j}} \frac{V_{j}-V_{k}}{R_{j k}}=0, \quad \forall j \in \mathcal{N} \backslash \mathcal{L},
$$

where $\mathcal{N} \backslash \mathcal{L}$ denotes the set of all non-load buses, $\mathcal{N}_{k} \subset \mathcal{N}$ denotes the set of all neighboring buses of bus $k$, and $R_{i k}$ denotes the line resistance between buses $i$ and $k$. The equalities in (2) and (3) are the fundamental equations to understand DC distribution systems and DC microgrids. Note that, in general, calculating the line resistance $R_{i k}$ can be difficult in practice. Interested readers can refer to [25] for more details on how to calculate the line resistance in distribution networks using catalogue data and synchronous measurements. Other papers that similarly consider line resistance in the context of DC distribution networks include [26] and [27].

From (2), at each load bus $i \in \mathcal{L}$ we have

$$
\begin{aligned}
\frac{V_{i}}{R_{i}} & =\frac{V_{i}^{s}-V_{i}}{R_{i}^{s}}+\sum_{k \in \mathcal{N}_{i}} \frac{V_{k}-V_{i}}{R_{i k}} . \\
& \Rightarrow \quad R_{i}=V_{i} /\left(\frac{V_{i}^{s}-V_{i}}{R_{i}^{s}}+\sum_{k \in \mathcal{N}_{i}} \frac{V_{k}-V_{i}}{R_{i k}}\right) .
\end{aligned}
$$

Also from (3), at each non-load bus $j \in \mathcal{N} \backslash \mathcal{L}$, we have

$$
\frac{V_{j}-V_{j}^{s}}{R_{j}^{s}}+\sum_{k \in \mathcal{N}_{j}} \frac{V_{j}}{R_{j k}}-\sum_{k \in \mathcal{N}_{j}, k \notin \mathcal{L}} \frac{V_{k}}{R_{j k}}=\sum_{k \in \mathcal{N}_{j}, k \in \mathcal{L}} \frac{V_{k}}{R_{j k}} .
$$

The left hand side in (5) is a linear combination of variables $V_{j}$ for all $j \in \mathcal{N} \backslash \mathcal{L}$; while the right hand side in (5) is a linear combination of variables $V_{i}$ for all $i \in \mathcal{L}$. Therefore, the system of $|\mathcal{N}|-|\mathcal{L}|$ equations in (5) can be solved to obtain the voltages $V_{j}$ for all $j \in \mathcal{N} \backslash \mathcal{L}$ in terms of voltages $V_{i}$ for all $i \in \mathcal{L}$. More specifically, from (5) we can derive:

$$
V_{j}=\sum_{i \in \mathcal{L}} a_{j i} V_{i}+b_{j}, \quad \forall j \in \mathcal{N} \backslash \mathcal{L},
$$

where parameters $a_{j i}$ and $b_{j}$ are constant. For example, consider the four-bus DC power system in Fig. 2(b). Here, $\mathcal{L}=\{1,3\}$ and $\mathcal{N} \backslash \mathcal{L}=\{2,4\}$. It can be shown that

$$
\begin{aligned}
& a_{21} \triangleq 1 /\left(1+R_{12} / R_{23}+R_{12} / R_{2}^{s}\right), \\
& a_{23} \triangleq 1 /\left(1+R_{23} / R_{12}+R_{23} / R_{2}^{s}\right), \\
& b_{2} \triangleq V_{2}^{s} /\left(1+R_{2}^{s} / R_{12}+R_{2}^{s} / R_{23}\right) .
\end{aligned}
$$

Parameters $a_{41}, a_{4,3}$, and $b_{4}$ can be derived similarly.

\section{B. Power Electronics Loads}

By replacing (6) in (4), at each load bus $i \in \mathcal{L}$, we have

$$
\begin{array}{r}
R_{i}=V_{i} /\left(\frac{V_{i}^{s}-V_{i}}{R_{i}^{s}}-\sum_{k \in \mathcal{N}_{i}} \frac{V_{i}}{R_{i k}}+\sum_{k \in \mathcal{N}_{i}, k \in \mathcal{L}} \frac{V_{k}}{R_{i k}}\right. \\
\left.+\sum_{k \in \mathcal{N}_{i}, k \notin \mathcal{L}}\left(\sum_{l \in \mathcal{L}} a_{k l} V_{l}+b_{k}\right) / R_{i k}\right) .
\end{array}
$$

The above equation expresses the variable resistor of each power electronics load in terms of the voltages at all load buses. Therefore, using (8), together with the rest of the system model in this section, we can formulate different objective functions in terms of bus voltages, which can be adjusted by changing the effective resistance of converters / rectifiers. 


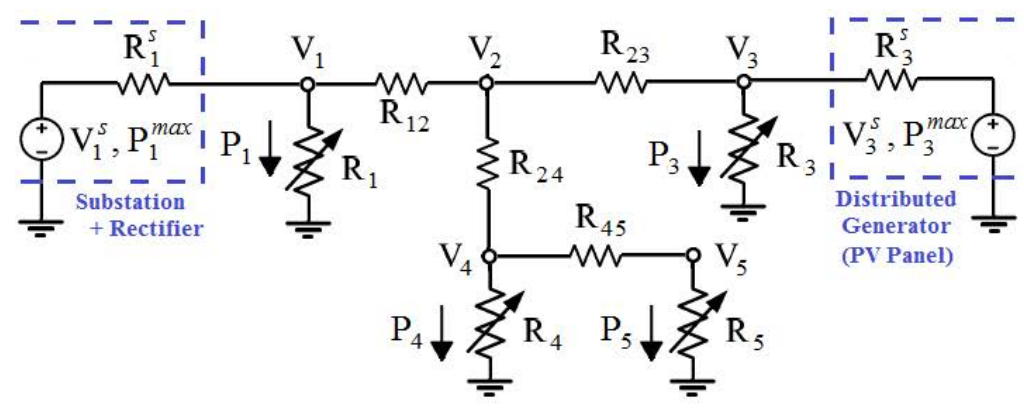

(a)

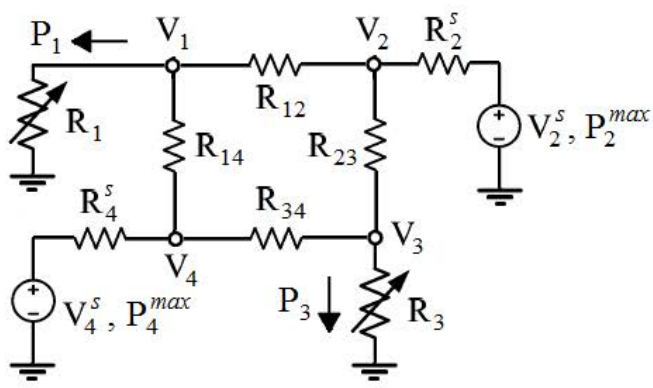

(b)

Fig. 2. Two examples for DC power systems: (a) A five-bus radial power distribution network with four power electronics loads. (b) A four-bus DC microgrid with two power electronics loads. Demand response is done by changing the variable resistors for the participating power electronics loads.

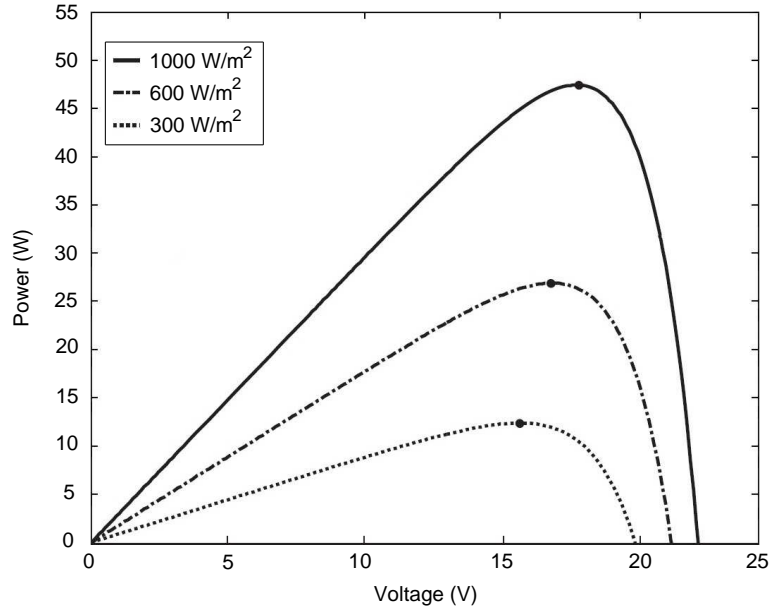

Fig. 3. The characteristics curves of a solar panel for three irradiance levels. The MPPT solution is shown by a solid point on each curve [29].

\section{Solar Panels}

The system model explained above can support distributed generation (DG), where PV panels are connected to the DC distribution network or DC microgrid. Each PV panel is equipped with a constant voltage (CV) control mechanism [28] to achieve maximum power point tracking (MPPT). Therefore, like a conventional generator, a PV panel at bus $i$ is modeled as a constant voltage DC source. However, the values of $V_{i}^{s}$ and $P_{i}^{\max }$ must be updated based on the solar irradiance level. That is, given the irradiance level, the CV-based MPPT control mechanism adjusts the values of $V_{i}^{s}$ and $P_{i}^{\max }$ according to the voltage-power characteristics curves of the PV panel [29], as shown in Fig. 3. For example, when the irradiance level is $1000 \mathrm{~W} / \mathrm{m}^{s}$ then $V_{i}^{s}=17.8 \mathrm{~V}$ and $P_{i}^{\max }=47.4 \mathrm{~W}$. Once the proposed optimal demand response method is implemented, whenever the irradiance level changes during the day, the users' power electronics loads are adjusted to control their power consumption in order to achieve the best overall system performance across the DC distribution network. In practice, such adjustment can be done on a periodic basis. Two examples for daily changes in the solar irradiance are shown in Fig. 4 based on the experimental measurements in [30].
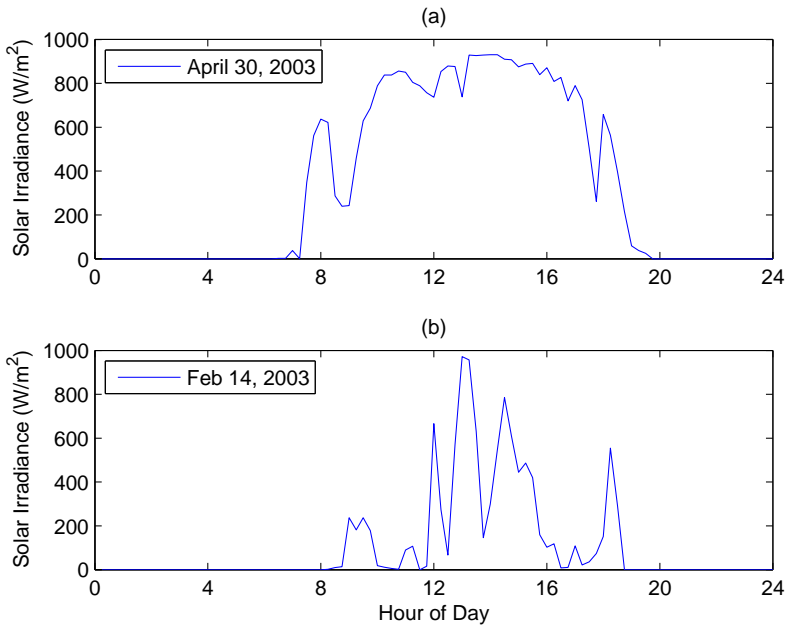

Fig. 4. Two examples for daily solar irradiance in Abilene, TX based on measurements every 15 minutes: (a) A mostly sunny day. (b) A cloudy day.

\section{Optimal Demand Response}

The focus of the proposed demand response paradigm in DC distribution networks is to treat power converter as power buffer and adjust the variable effective resistance of power electronics loads to assure DC power system reliability and efficiency. The three key intended characteristics of this demand response paradigm are as follows:

- The amount of power drawn from the DC power source at each source bus should not exceed its supply limit.

- The power electronics loads should support their intended demand response solutions based on their characteristics.

- The power should be delivered fairly among the loads at different locations of the DC distribution network.

First, consider the power draw limits from DC sources. For the DC power source at each source bus $k$, the amount of current drawn can be calculated as $\left(V_{k}^{s}-V_{k}\right) / R_{k}^{s}$. Therefore, the power limit requirement can be formulated as $V_{k}^{s}\left(V_{k}^{s}-\right.$ $\left.V_{k}\right) / R_{k}^{s} \leq P_{k}^{\max }$. In other words, it is required to have

$$
V_{k} \geq V_{k}^{s}-P_{k}^{\max } R_{k}^{s} / V_{k}^{s}, \quad \forall k \in \mathcal{N} .
$$

From (6), one can rewrite the above constraints as

$$
V_{i} \geq e_{i}, \quad \forall i \in \mathcal{L},
$$


and

$$
\sum_{i \in \mathcal{L}} a_{j i} V_{i} \geq e_{j}, \quad \forall j \in \mathcal{N} \backslash \mathcal{L},
$$

where for each $i \in \mathcal{L}$, we have $e_{i}=V_{i}^{s}-P_{i}^{\max } R_{i}^{s} / V_{i}^{s}$ and for each $j \in \mathcal{N} \backslash \mathcal{L}$, we have $e_{j}=V_{j}^{s}-P_{j}^{\max } R_{j}^{s} / V_{j}^{s}-b_{j}$.

Next, using (8), at each load bus $i \in \mathcal{L}$, the power delivered to the power electronics load is calculated as

$$
P_{i}=\frac{V_{i}^{2}}{R_{i}}=V_{i}\left(\sum_{j \in \mathcal{L}} c_{i j} V_{j}+d_{i}\right),
$$

where

$$
\begin{gathered}
c_{i i}=\sum_{k \in \mathcal{N}_{i}, k \notin \mathcal{L}} \frac{a_{k i}}{R_{i k}}-\sum_{k \in \mathcal{N}_{i}} \frac{1}{R_{i k}}-\frac{1}{R_{i}^{s}}, \\
d_{i}=\frac{V_{i}^{s}}{R_{i}^{s}}+\sum_{k \in \mathcal{N}_{i}, k \notin \mathcal{L}} \frac{b_{k}}{R_{i k}},
\end{gathered}
$$

and for each load bus $k \in \mathcal{L} \backslash\{i\}$, we have

$$
c_{i k}=\sum_{l \in \mathcal{N}_{i}, l \notin \mathcal{L}} \frac{a_{l k}}{R_{i k}}+\frac{1_{k \in \mathcal{N}_{i}}}{R_{i k}} .
$$

In (15), $1_{k \in \mathcal{N}_{i}}$ is an indicator function. That is, if $k \in \mathcal{N}_{i}$, then $1_{k \in \mathcal{N}_{i}}=1$; otherwise, $1_{k \in \mathcal{N}_{i}}=0$.

Whether a power electronics load can support a demand response solution depends on the characteristics of its DC-DC converter. Let $D_{i}^{\min }$ and $D_{i}^{\max }$ denote the minimum and the maximum permissible duty cycles that the DC-DC converter at load bus $i$ can support. It is required that

$$
D_{i}^{\min } \leq D_{i} \leq D_{i}^{\max }, \quad \forall i \in \mathcal{L} .
$$

From (1), one can rewrite the above constraints as

$$
R_{i}^{\min } \leq R_{i} \leq R_{i}^{\max }, \quad \forall i \in \mathcal{L},
$$

where

$$
\begin{aligned}
& R_{i}^{\min }=\left(1-D_{i}^{\max }\right)^{2} R_{i}^{o}, \\
& R_{i}^{\max }=\left(1-D_{i}^{\min }\right)^{2} R_{i}^{o} .
\end{aligned}
$$

Here, $R_{i}^{o}$ denotes the internal resistance of the actual end load at bus $i$. From (8) and (17) and after reordering the terms, the following constraints must hold on the voltages at load buses:

$$
\sum_{k \in \mathcal{L}} m_{i k} V_{k} \geq f_{i}, \quad \forall i \in \mathcal{L},
$$

and

$$
\sum_{k \in \mathcal{L}} n_{i k} V_{k} \leq g_{i}, \quad \forall i \in \mathcal{L},
$$

where

$$
\begin{aligned}
& m_{i i}=1-c_{i i} R_{i}^{\min }, \\
& n_{i i}=1-c_{i i} R_{i}^{\max },
\end{aligned}
$$

and for each $k \in \mathcal{L} \backslash\{i\}$, we have

$$
\begin{aligned}
& m_{i k}=c_{i k} R_{i}^{\min }, \\
& n_{i k}=c_{i k} R_{i}^{\max } .
\end{aligned}
$$

For each $i \in \mathcal{L}$, we have

$$
\begin{aligned}
f_{i} & =\frac{R_{i}^{\min }}{R_{i}^{s}} V_{i}^{s}+\sum_{k \in \mathcal{N}_{i}, k \notin \mathcal{L}} \frac{R_{i}^{\min }}{R_{i k}} b_{k}, \\
g_{i} & =\frac{R_{i}^{\max }}{R_{i}^{s}} V_{i}^{s}+\sum_{k \in \mathcal{N}_{i}, k \notin \mathcal{L}} \frac{R_{i}^{\max }}{R_{i k}} b_{k} .
\end{aligned}
$$

Depending on the load characteristics, the constraints in (20) and (21) may sometimes be relaxed. For example, if $D_{i}^{\max } \approx$ 1 , then $R_{i}^{\min }=0$ and constraint (20) becomes $V_{i} \geq 0$.

The optimal DR problem can now be formulated for DC distribution networks. Here, the notion of proportional fairness from utility theory [31] is used. Proportional fairness is achieved in power delivered to users if one can maximize:

$$
\prod_{i \in \mathcal{L}} P_{i} .
$$

The expression in (28) is directly linked to the concept of Nash bargaining solution in Cooperative Game Theory [32]. Intuitively, any $P_{i}$ that is too low can significantly lower the overall product in (28). Therefore, by maximizing (28), no single load suffers from a power draw that is too low, assuring fairness. Since the logarithm function is a monotonically increasing function, maximizing (28) is equivalent to maximizing [33]:

$$
\log \left(\prod_{i \in \mathcal{L}} P_{i}\right)=\sum_{i \in \mathcal{L}} \log \left(P_{i}\right) .
$$

From this, together with (10)-(12), to assure fair power delivery to users while observing the power limits of DC sources, one must solve the following optimization problem:

$$
\begin{aligned}
\underset{V_{i} \geq e_{i}, \forall i \in \mathcal{L}}{\operatorname{maximize}} & \sum_{i \in \mathcal{L}} \log \left(V_{i}\right)+\sum_{i \in \mathcal{L}} \log \left(\sum_{j \in \mathcal{L}} c_{i j} V_{j}+d_{i}\right) \\
\text { subject to } & \sum_{i \in \mathcal{L}} a_{j i} V_{i} \geq e_{j}, \quad \forall j \in \mathcal{N} \backslash \mathcal{L}, \\
& \sum_{k \in \mathcal{L}} m_{i k} V_{k} \geq f_{i}, \quad \forall i \in \mathcal{L}, \\
& \sum_{k \in \mathcal{L}} n_{i k} V_{k} \leq g_{i}, \quad \forall i \in \mathcal{L} .
\end{aligned}
$$

The following theorem summarizes the key characteristics and the importance of the above optimization problem.

Theorem 1: (a) Problem (30) is a convex optimization problem. (b) Given $V_{i}^{*}$ for all $i \in \mathcal{L}$ as the optimal solution of (30), the optimal values for variable resistors are obtained using (8). The optimal duty cycles for the switch-mode converters can be selected accordingly, e.g., using (1) for a boost converter.

Proof: To prove part (a), it is noted that since logarithm is a concave function and the expression inside the parenthesis for the second logarithmic term in the objective function in (30) is affine, the objective function in optimization problem (30) is concave. From this, together with the fact that all constraints are linear, the maximization problem in (30) is a convex program. The proof of part (b) is evident.

From Theorem 1, if direct load control (DLC) is possible, then optimal demand response is achieved in four steps. First, 
collect all needed data from each load, bus, and link in the DC distribution network and send them to an aggregator. Second, solve problem (30) using standard convex programming tools. Third, announce the optimal effective resistance to each load that participates in demand response. Four, each load will adjust the duty cycle for its DC-DC converter accordingly.

\section{DECENTRALIZED IMPLEMENTATION}

If DLC is not feasible, then optimal demand response is done via pricing and a decentralized algorithm as we see next.

\section{A. Dual Decomposition}

From duality theory [22, Chapter 5], the Lagrangian associated with the primal optimization problem (30) becomes:

$$
\begin{aligned}
& L\left(\boldsymbol{V}_{\mathcal{L}}, \boldsymbol{\lambda}_{\mathcal{N} \backslash \mathcal{L}}, \boldsymbol{\mu}_{\mathcal{L}}, \boldsymbol{\rho}_{\mathcal{L}}\right)=\sum_{i \in \mathcal{L}} \log \left(V_{i}\right) \\
& +\sum_{i \in \mathcal{L}} \log \left(\sum_{j \in \mathcal{L}} c_{i j} V_{j}+d_{i}\right)-\sum_{j \in \mathcal{N} \backslash \mathcal{L}} \lambda_{j}\left(e_{j}-\sum_{k \in \mathcal{L}} a_{j k} V_{k}\right) \\
& -\sum_{i \in \mathcal{L}} \mu_{i}\left(f_{i}-\sum_{k \in \mathcal{L}} m_{i k} V_{k}\right)+\sum_{i \in \mathcal{L}} \rho_{i}\left(g_{i}-\sum_{k \in \mathcal{L}} n_{i k} V_{k}\right),
\end{aligned}
$$

where $\lambda_{j} \geq 0, \mu_{i} \geq 0$, and $\rho_{i} \geq 0$ are the Lagrange multipliers associated with the inequality constraints in (10), (20), and (21), respectively. We define the Lagrange dual function as

$$
\begin{aligned}
g\left(\boldsymbol{\lambda}_{\mathcal{N} \backslash \mathcal{L}}, \boldsymbol{\mu}_{\mathcal{L}}, \boldsymbol{\rho}_{\mathcal{L}}\right)=-\sum_{j \in \mathcal{N} \backslash \mathcal{L}} \lambda_{j} e_{j}-\sum_{i \in \mathcal{L}} \mu_{i} f_{i}+\sum_{i \in \mathcal{L}} \rho_{i} g_{i} \\
+\operatorname{maximum}_{V_{i} \geq e_{i}, \forall i \in \mathcal{L}} \sum_{i \in \mathcal{L}} \log \left(V_{i}\right) \\
+\sum_{i \in \mathcal{L}} \log \left(\sum_{j \in \mathcal{L}} c_{i j} V_{j}+d_{i}\right) \\
+\sum_{i \in \mathcal{L}}\left(\sum_{j \in \mathcal{N} \backslash \mathcal{L}} \lambda_{j} a_{j i}+\sum_{k \in \mathcal{L}} \mu_{k} m_{i k}-\rho_{k} n_{i k}\right) V_{i} .
\end{aligned}
$$

Finally, one can define the dual optimization problem as

$$
\underset{\lambda_{j} \geq 0, \forall j \in \mathcal{N} \backslash \mathcal{L}}{\operatorname{minimime}} g\left(\boldsymbol{\lambda}_{\mathcal{N} \backslash \mathcal{L}}\right)
$$

Recall from Theorem 1 that the primal optimization problem (30) is a convex program. From this, together with the fact that the linear constraints in (30) satisfy the Slater's condition, strong duality holds, and the duality gap is zero, and the Lagrange multipliers always exist [22, Section 5.2.3]. Therefore, if one can iteratively solve the maximization in (32) and the minimization in (33), then after convergence, the global optimal solution of the primal problem (30) will be achieved. In this regard, the coordinate ascent method [34, Section 3.2.4] can be used to solve problem (32) and the gradient method [22, Section 9.3] can be used to solve problem (33).

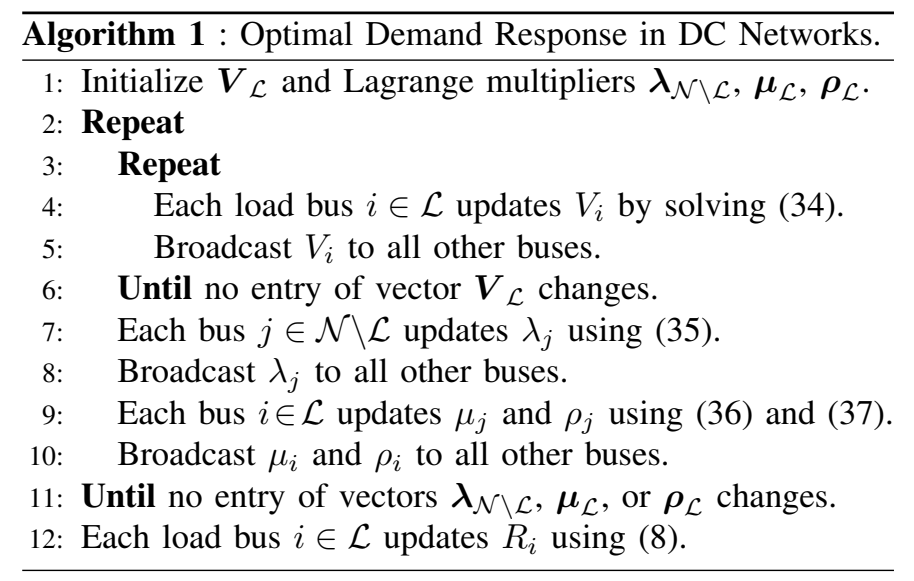

In order to solve problem (32) using the coordinate ascent method, one can fix all variables $V_{k}$ for $k \in \mathcal{L} \backslash\{i\}$, and then solve the optimization problem with respect to $V_{i}$ as follows:

$$
\begin{aligned}
\underset{V_{i} \geq e_{i}}{\operatorname{maximize}} & \sum_{i \in \mathcal{L}} \log \left(V_{i}\right)+\sum_{i \in \mathcal{L}} \log \left(\sum_{k \in \mathcal{L}} c_{i k} V_{k}+d_{i}\right) \\
& +\sum_{i \in \mathcal{L}}\left(\sum_{j \in \mathcal{N} \backslash \mathcal{L}} \lambda_{j} a_{j i}+\sum_{k \in \mathcal{L}} \mu_{k} m_{i k}-\rho_{k} n_{i k}\right) V_{i}
\end{aligned}
$$

This procedure is repeated for all $i \in \mathcal{L}$, leading to an iterative algorithm. Since problem (32) is convex, if the iterations are implemented in the form of a Gauss-Seidel algorithm, where users take turns, then the iterations are guaranteed to converge to the optimal solution of problem (32) [34, Proposition 2.5].

In order to solve problem (33) using the gradient method, at each iteration, we update the Lagrange multipliers as follows:

$$
\begin{aligned}
& \lambda_{j} \leftarrow\left[\lambda_{j}+\gamma\left(e_{j}-\sum_{k \in \mathcal{L}} a_{j k} V_{k}\right)\right]^{+}, \quad j \in \mathcal{N} \backslash \mathcal{L}, \\
& \mu_{i} \leftarrow\left[\mu_{j}+\sigma\left(f_{i}-\sum_{k \in \mathcal{L}} m_{i k} V_{k}\right)\right]^{+}, \quad i \in \mathcal{L}, \\
& \rho_{i} \leftarrow\left[\rho_{j}-\xi\left(g_{i}-\sum_{k \in \mathcal{L}} n_{i k} V_{k}\right)\right]^{+}, \quad i \in \mathcal{L},
\end{aligned}
$$

where $\gamma>0, \sigma>0$, and $\xi>0$ are stepsize parameters and $[z]^{+}=\max \{z, 0\}$. If the stepsize parameters are small enough or diminishing, then the convergence of the iterations in (35)-(37) to the solution of problem (33) is guaranteed [35].

We are now ready to introduce our proposed decentralized demand response scheme in Algorithm 1. There are two loops in this algorithm. The inner-loop solves problem (32) using the coordinated ascent method. The outer loop solves problem (33) using the gradient method. As we explained earlier, since the duality gap is zero, Algorithm 1 will converge to the optimal solution of problem (30), as long as the load bus voltages in Line 4 are updated sequentially and the step size to update the Lagrange multipliers in Line 7 is small enough or diminishing. 


\section{B. Pricing Interpretation}

Consider the local problem (34) that each load bus (or user) must solve when Algorithm 1 is implemented. From (12), and after reordering the terms, it can be shown that problem (34) is equivalent [22, Section 4.1.3] to the following problem:

$$
\underset{V_{i} \geq e_{i}}{\operatorname{maximize}} \log \left(P_{i}\right)+\alpha_{i} V_{i}+\sum_{k \in \mathcal{L} \backslash\{i\}} \log \left(c_{k i} V_{i}+\beta_{i k}\right)
$$

where

$$
\alpha_{i}=\sum_{j \in \mathcal{N} \backslash \mathcal{L}} \lambda_{j} a_{j i}+\sum_{k \in \mathcal{L}} \mu_{k} m_{i k}-\sum_{k \in \mathcal{L}} \rho_{k} n_{i k}
$$

and for each $k \in \mathcal{N} \backslash\{i\}$, we have

$$
\beta_{i k}=\sum_{l \in \mathcal{L} \backslash\{i\}} c_{k l} V_{l}+d_{k} .
$$

As far as solving problem (38) is concerned, $\alpha_{i}$ and $\beta_{i k}$ are constant. They can take both positive and negative values.

The objective function in (38) can be interpreted as follows. The first term, $\log \left(P_{i}\right)$, can be seen as user $i$ 's monotonicallyincreasing and concave utility function that quantifies user $i$ 's level of satisfaction when it draws power $P_{i}$, c.f. [36], [37]. The second term, $\alpha_{i} V_{i}$, is for voltage regulation. If $\alpha_{i}<0$, then user $i$ is encouraged to reduce its voltage. If $\alpha_{i}>0$, then user $i$ is encouraged to increase its voltage. Finally, the third term, $\sum_{k \in \mathcal{L} \backslash\{i\}} \log \left(c_{k i} V_{i}+\beta_{i k}\right)$, can enforce fairness. The higher the value of $\beta_{i k}$, the stronger user $i$ is encouraged to regulate its voltage and allow more power delivery to user $k$.

Based on the discussion above, one can interpret $\alpha_{i}$ as a voltage regulating price and $\beta_{i k}$ as a fairness enforcement price. From (39), the voltage regulating price depends on $\lambda_{j}$, i.e., whether any DC source at a non-load bus $j$ has reached its power delivery limit, and also on $a_{j i}$, i.e., the way that the operation of the variable resistor load at load bus $i$ may affect the voltage at the terminal of a DC source at a nonload bus $j$. From (40), the fairness enforcement price depends on the amount of current that user $k$ can draw from the DC distribution network if $R_{i} \rightarrow 0$ and, accordingly, $V_{i} \rightarrow 0$.

\section{Performance Evaluation}

In this section, the performance of the proposed optimal demand response framework for DC distribution networks is assessed. We examine both centralized and decentralized designs. All parameters are for a per unit system with base voltage of $380 \mathrm{~V}$ DC and base power of $10 \mathrm{~kW}$ [38]. Unless stated otherwise, it is assumed that $R_{i}^{\min }=0$ and $R_{i}^{\max } \gg 1$.

\section{A. Achieving Efficiency and Fairness}

First, consider the radial distribution network in Fig. 2(a) with $R_{12}=0.01, R_{23}=0.03, R_{24}=0.01, R_{45}=0.01$, $R_{1}^{s}=0.01, R_{3}^{s}=0.01, V_{1}^{s}=1, V_{3}^{s}=1, P_{1}^{\max }=4$, and $P_{3}^{\max }=2.5$. In presence of the distributed generator at bus 3 , the optimal values for the variable resistors are $R_{1}^{*}=0.4921$, $R_{3}^{*}=0.7404, R_{4}^{*}=0.5563$, and $R_{5}^{*}=0.5565$. The amount of power delivered to each load is shown in Fig. 5(a). At optimality, both DC sources reach their power limits $P_{1}^{\max }$
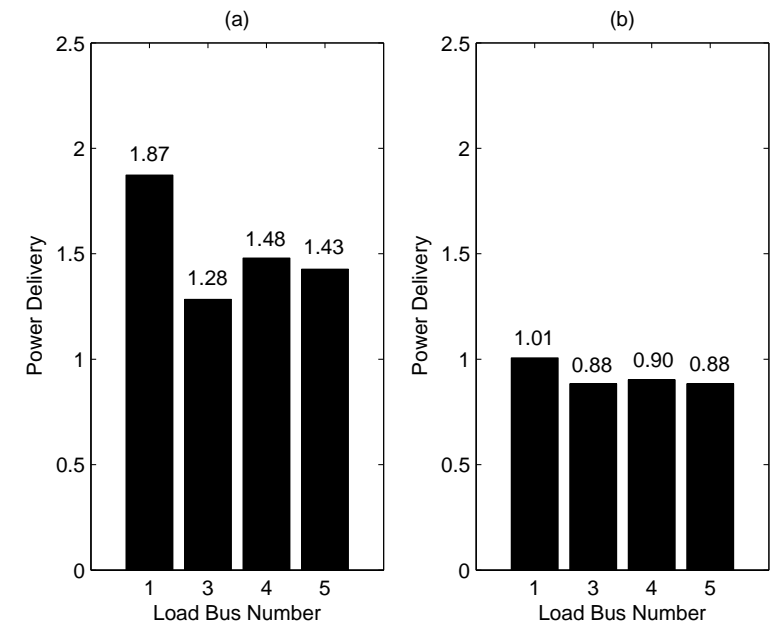

Fig. 5. Power delivery to end users based on the solution of problem (30) in the radial power distribution network of Fig. 2(a) with four power electronics loads: (a) With distributed generation. (b) Without distributed generation.

and $P_{3}^{\max }$. Next, assume that the distributed generator at bus 3 is disconnected from the network. The optimal values for the variable resistors are updated accordingly and we have $R_{1}^{*}=0.9159, R_{3}^{*}=0.9192, R_{4}^{*}=0.9189, R_{5}^{*}=0.9190$. The amount of power delivered to each load is shown in Fig. 5(b). One can see that the proposed design can maintain both efficiency and fairness under different grid conditions.

\section{B. Different Random Scenarios}

In order to have a base for performance comparison, in this section, we consider the utility-based demand response design in [36]. Note that, if the utility functions are logarithmic, then the objective function of the utility maximization problem in [36] becomes identical to the objective function of the optimization problem in equation (30) of this paper. However, since the design in [36] does not take into account the details of the underlying physical power system, if the design guidelines in [36] are followed, then all users that are connected to the same feeder and have the same utility functions will have the same effective resistance in their loads. For example, for the radial DC distribution network in Fig. 2(a), [36] results in

$$
R_{1}=R_{3}=R_{4}=R_{5} .
$$

The proposed method here is compared with the design approach in [36] based on (41), and the results are shown in Fig. 6(a). Here, 100 different scenarios are considered by randomly choosing parameters $R_{12}, R_{23}, R_{24}, R_{45}, R_{1}^{s}, R_{3}^{s}$, $P_{1}^{\max }$, and $P_{3}^{\max }$. The resulting proportional fairness objective values are compared for each scenario. To ensure a fair comparison, the best possible performance when (41) holds is considered for each scenario. This gives an upper bound for the design performance in [36]. Such upper bound is then compared with the proposed design in this paper. The details of such comparison for scenario number 1 are shown in Fig. 6(b), as an example. On average, and across all 100 random scenarios, the proportional fairness objective value increases by $11.2 \%$ when the proposed design is implemented compared 
(a)
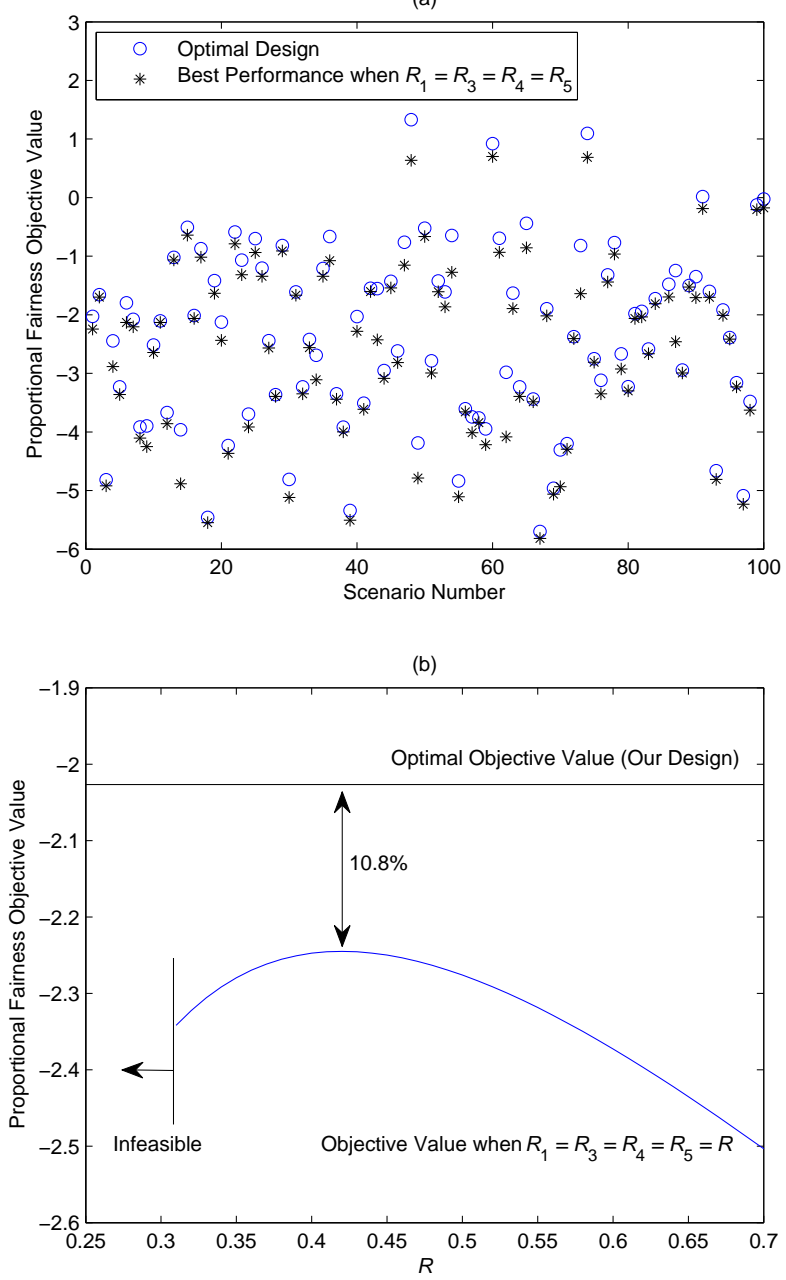

Fig. 6. Performance comparison between the proposed optimal design and the case where $R_{1}=R_{3}=R_{4}=R_{5}$ in the radial power distribution network of Fig. 2(a) with four power electronics loads: (a) Comparison across 100 random scenarios. (b) Detailed comparison based on scenario number 1 .

with the best performance possible when (41) holds. Given that the proposed method in this paper and the method in [36] have identical objective functions for logarithmic utilities, the advantage of our proposed design is evident. Of course, we must note that, while the design in [36] is general and can accommodate different choices of utility functions beyond logarithmic utilities, the proposed method in this paper, however, is specifically designed for logarithmic utility functions towards achieving proportional fairness.

\section{Decentralized Design and Pricing}

Consider the DC microgrid in Fig. 2(b), where $R_{12}=0.01$, $R_{14}=0.03, R_{23}=0.01, R_{34}=0.01, R_{2}^{s}=0.01, R_{4}^{s}=0.01$, $V_{2}^{s}=1, V_{4}^{s}=1, P_{2}^{\max }=4$, and $P_{4}^{\max }=3$. Note that, the two DC power sources are connected to non-load buses. The power draw constraints in this example are

$$
0.3333 V_{1}+0.3333 V_{3} \geq 0.6267,
$$

and

$$
0.1429 V_{1}+0.4286 V_{3} \geq 0.5414 .
$$
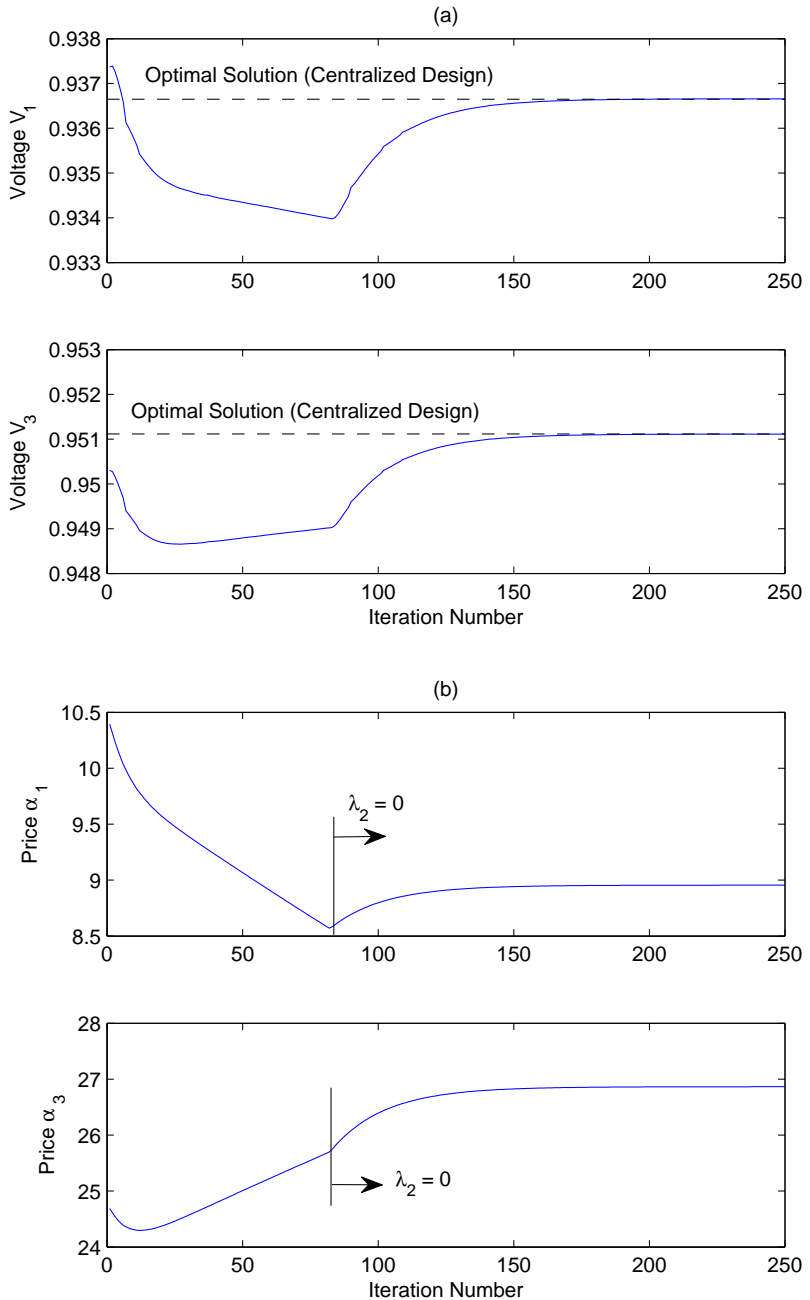

Fig. 7. Performance evaluation of distributed Algorithm 1 for the DC microgrid in Fig. 2(b): (a) The trends of the voltages at load buses 1 and 3. (b) The trends of the voltage regulation prices at load buses 1 and 3 .

The simulation results when we run Algorithm 1 are shown in Fig. 7. We can see that the distributed design results in the exact optimal performance as in the centralized design. In particular, both $V_{1}$ and $V_{3}$, i.e., the voltages at the load buses, converge to their optimal values as shown in Fig. 7(a). The voltage regulation price signals are shown in Fig. 7(b). At optimality, only the DC source at bus 4 reaches its power limit $P_{4}^{\max }$. Therefore, at steady state, we have $\lambda_{4}>0$ while $\lambda_{2}=$ 0 . Note that, only the iterations in the outer loop of Algorithm 1 are shown here. Typically, the inner loop converges within 3 or 4 iterations. Constraints (20) and (21) are not binding.

It is interesting to compare Fig. 7 with the similar results in Fig. 5 of the conference version of this work in [23]. Here, we have chosen a large step size $\gamma=100$ for the Lagrange multiplier update equation in (35). This has resulted in an order of magnitude faster convergence in Fig. 7, compared to Fig. 5 in [23].

\section{Impact of Time-Varying Renewable Generation}

Next, the variable resistors are adjusted in response to changes in solar irradiance. The system setup is the same as 
(a)

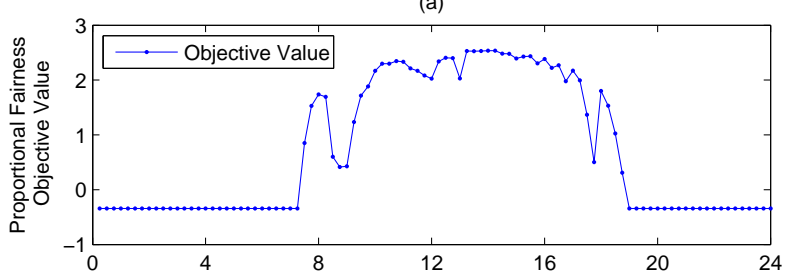

(b)

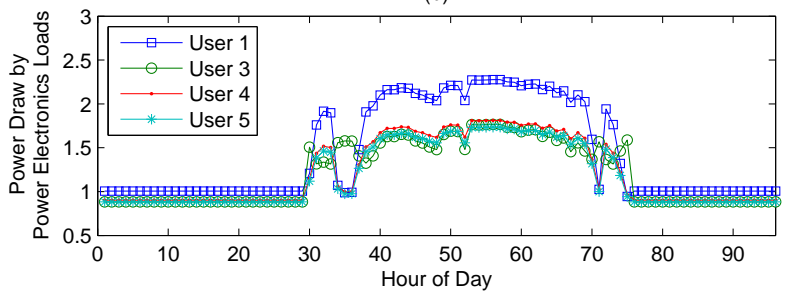

Fig. 8. Simulation results when the variable resistors in Fig. 2(a) are updated every 15 minutes based on the irradiance data in Fig. 4(a): (a) Proportional fairness objective value. (b) Power draw by power electronics loads.

that in Section V-A. However, the power and voltage at the PV panel are updated every 15 minutes using the data in Fig. 4(a). At $600 \mathrm{~W} / \mathrm{m}^{2}$ solar irradiance, the delivered power ad voltage are $P_{3}^{\max }=2.5 \mathrm{MW}$ and $V_{3}^{s}=380 \mathrm{~V}$ DC, respectively. The values of $P_{3}^{\max }$ and $V_{3}^{s}$ at other irradiance levels are adjusted based on the curves in Fig. 3, with interpolation when necessary. The results are shown in Fig. 8. We can see that the proportional fairness objective value in Fig. 8(a) resembles the daily irradiance curve in Fig. 4(a). At each point, the optimal objective value of problem (30) is achieved based on the PV panel conditions at the corresponding time of day. The amount of power draw by each end user is also adjusted accordingly at each point as we can see in Fig. 8(b).

\section{E. Impact of DC-DC Converter Duty Cycle Limitations}

So far, all DC-DC converters that serve the power electronics loads are assumed ideal and support any duty cycle. In other words, at each load bus $i \in \mathcal{L}$, we have $R_{i}^{\text {min }}=0$ and $R_{i}^{\min } \gg 1$. Next, we relax this assumption and study the impact of changing the permissible range for the duty cycles of the DC-DC converters. We assume that at each load bus $i \in \mathcal{L}$, we have $D_{i}^{\min }=0.2$ and $D_{i}^{\max }$ varies from 0.3 to 0.7. The results for the proportional fairness objective value are shown in Fig. 9. In all cases, the objective value is much higher when the duty cycles are less restricted. More flexible DC-DC converters are particularly beneficial in presence of PV panels. Here, the solar irradiance data for the sunny day and the cloudy day come from Figs. 4(a) and (b), respectively.

\section{CONCLUSIONS AND Future WORK}

Given the great benefits of DC distribution networks and because of the importance of demand response in the future smart grid, in this paper, the first steps were taken towards designing demand response programs for DC distribution systems. In this regard, the recent advancements in power electronics were combined with techniques from optimization theory to develop

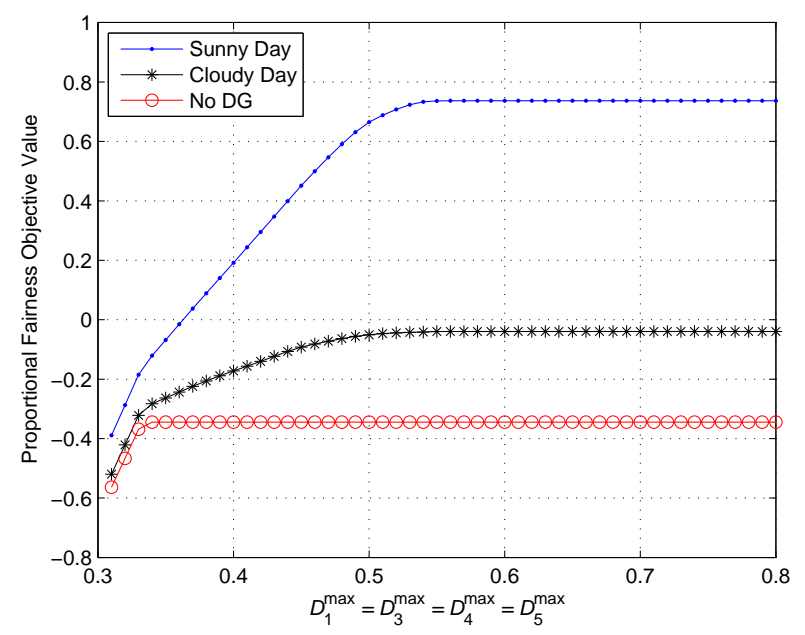

Fig. 9. The impact of changing permissible range for duty cycles of power electronics loads. We have $D_{i}^{\min }=0.2$ and $D_{i}^{\max }$ varies from 0.3 to 0.8 . As expected, a wider range for the duty cycle improves the objective value.

an optimization-based DC demand response foundation to ensure efficient and fair power system operation. It was shown that the formulated optimization problem is convex and, therefore, tractable. Both centralized and decentralized designs were examined. Furthermore, a pricing mechanism was developed to enforce optimal DC demand response in a distributed fashion. Various simulation results showed that the proposed design can maximize the efficiency of renewable and distributed generation resources such as photovoltaic panels.

The results in this paper can be extended in several directions. For example, design objective functions other than proportional fairness can be examined. Other constraints, e.g., with respect to DC distribution loss, can also be considered. Other MPPT models such as constant power systems can be considered for PV panels. Data collection and communications among power converters can be realized using different networking protocols depending on missions and applications. In particular, the impact of using different data transmission techniques such as wireless mesh networks, cellular, and power lines carriers can be investigated. The proposed design may also be evaluated using hardware implementation.

\section{REFERENCES}

[1] K. Hamilton and N. Gulhar, "Taking demand response to the next level," IEEE Power and Energy Magazine, vol. 8, no. 3, pp. 60-65, May 2010.

[2] G. Strbac, "Demand side management: Benefits and challenges," Energy Policy, vol. 36, no. 12, pp. 4419-4426, Dec. 2008.

[3] H. Mohsenian-Rad and A. Leon-Garcia, "Optimal Residential Load Control with Price Prediction in Real-Time Electricity Pricing Environments," IEEE Trans. on Smart Grid, vol. 1, pp. 120-133, Sept. 2010.

[4] A. Molderink, V. Bakker, J. L. Hurink, and G. J. M. Smit, "Comparing demand side management approaches," in Proc. of IEEE PES ISGT'12, Washington, DC, Jan. 2010.

[5] H. Mohsenian-Rad, V. Wong, J. Jatskevich, R. Schober, and A. LeonGarcia, "Autonomous Demand Side Management Based on GameTheoretic Energy Consumption Scheduling for the Future Smart Grid," IEEE Trans. on Smart Grid, vol. 1, no. 3, pp. 320-331, Dec. 2010.

[6] Z. Baharlouei, M. Hashemi, H. Narimani, and H. Mohsenian-Rad, "Achieving Optimality and Fairness in Autonomous Demand Response: Benchmarks and Billing Mechanisms," IEEE Trans. on Smart Grid, vol. 4, no. 2, pp. 968-975, June 2013. 
[7] J. Medina, N. Muller, and I. Roytelman, "Demand Response and Distribution Grid Operations: Opportunities and Challenges," IEEE Trans. on Smart Grid, vol. 1, no. 2, pp. 193-198, Sept. 2010.

[8] N. Li, L. Chen, and S. Low, "Demand response in radial distribution networks," in Proc. of the IEEE Systems and Computers Asilomar Conference on Signals, Pacific Grove, CA, Nov. 2012.

[9] S. Mohagheghi, J. Stoupis, Z. Wang, Z. i, and H. Kazemzadeh, "Demand response architecture: Integration into the distribution management system," in Proc. of IEEE International Conference on Smart Grid Communications, Gaithersburg, MD, Oct. 2010.

[10] E. L. Zivi, "Integrated shipboard power and automation control challenge problem," in Proc. of the IEEE Power Engineering Society Summer Meeting, Chicago, IL, July 2002.

[11] M. E. Baran and N. R. Mahajan, "DC distribution for industrial systems: Opportunities and challenges," IEEE Trans. on Industrial Applications, vol. 39, no. 6, pp. 1596-1601, Nov. 2003.

[12] H. Kakigano, Y. Miura, and T. Ise, "Low voltage bipolar type DC microgrid for super high quality distribution," IEEE Trans. on Power Electronics, vol. 25, no. 12, pp. 3066-3075, Nov. 2010.

[13] M. Brenna, E. Tironi, and G. Ubezio, "Proposal of a local de distribution network with distributed energy resources," in Proc. of the IEEE ICHQP, Lake Placid, NY, 2004.

[14] D. Salomonsson and A. Sannino, "Low-Voltage DC Distribution System for Commercial Power Systems With Sensitive Electronic Loads," IEEE Trans. on Power Delivery, vol. 22, no. 3, pp. 1620-1627, July 2007.

[15] R. S. Balog, W. Weaver, and P. T. Krein, "The Load as an Energy Asset in a Distributed DC SmartGrid Architecture," IEEE Trans. on Smart Grid, vol. 3, no. 1, pp. 253-260, Mar. 2012.

[16] A. Kwasinski and C. N. Onwuchekwa, "Dynamic behaviour and stablization of dc microgrids with instantaneous constant-power loads," IEEE Trans Power Electronics, vol. 26, pp. 822-834, Mar. 2011.

[17] H. Ikebe, "Power systems for telecommunications in the IT age," in Proc. of IEEE INTELEC, Yokohama, Japan, Oct. 2003.

[18] A. Kwasinski and C. N. Onwuchekwa, "Quantitate evaluation of dc microgrids availability: effects of system architecture and converter topology design choices," IEEE Trans Power Electronics, vol. 26, pp. 835-851, Mar. 2011.

[19] W. Weaver, "Dynamic Energy Resource Control of Power Electronics in Local Area Power Networks," IEEE Trans. on Power Electronics, vol. 26, no. 3, pp. 852-859, Mar. 2011.

[20] A. Mohamed and O. Mohammed, "Power flow control in dc distribution systems," in Proc. of the IEEE NAPS, Arlington, TX, 2010.

[21] W. Weaver and P. T. Krein, "Game-Theoretic Control of Small-Scale Power Systems," IEEE Trans. on Power Delivery, vol. 24, no. 3, pp. $1560-1567$, July 2009.

[22] S. Boyd and L. Vandenberghe, Convex Optimization. New York, NY: Cambridge University Press, 2004.

[23] H. Mohsenian-Rad and A. Davoudi, "Optimal demand response in dc distribution networks," in Proc. of IEEE International Conference on Smart Grid Communications, Vancouver, BC, Oct. 2013.

[24] R. W. Erickson and D. Maksimovic, Fundamentals of Power Electronics. New York, NY: Kluwer Academic Publishers, 2004

[25] A. M. Dumitrescu, I. Florea, M. Naumof, and M. Calin, "Model validation of distribution feeders using synchronous measurements," in Proc. of IEEE International Instrumentation and Measurement Technology Conference, Graz, Austria, May 2012.

[26] S. Grillo, V. Musolino, L. Piegari, and E. Tironi, "A control strategy for optimizing the power flows supplied by two different storage units," in Proc. of IEEE PowerTech, Trondheim , Norway, June 2011.

[27] A. Mohamed and O. Mohammed, "Connectivity of dc microgrids involving sustainable energy sources," in Proc. of IEEE Industry Applications Society Annual Meeting, Orlando, FL, Oct. 2011.

[28] G. J. Yu, Y. S. Jung, J. Y. Choi, I. Choy, J. H. Song, and G. S. Kim, "A novel two-mode MPPT control algorithm based on comparative study of existing algorithms," in Proc. of IEEE Photovoltaic Specialists Conference, New Orleans, LA, May 2002.

[29] T. Esram and P. L. Chapman, "Comparison of photovoltaic array maximum power point tracking techniques," IEEE Trans. on Energy Conversion, vol. 22, no. 2, pp. 439-449, June 2007.

[30] Solar Energy Laboratory at The University of Texas at Austin, "Texas Solar Radiation Database," http://www.me.utexas.edu/ solarlab/tsrdb/tsrdb.html, July 2013.

[31] F. Kelly, A. Maulloo, and D. Tan, "Rate control for communication networks: Shadow price proportional fairness and stability," Journal of Operations Research, vol. 49, pp. 237-252, Jan. 1998

[32] D. Fudenberg and J. Tirole, Game Theory. The MIT Press, 1991.
[33] L. Massoulie, "Structural properties of proportional fairness: Stability and insensitivity," Annals of Applied Probability, vol. 17, no. 3, pp. 809-839, Mar. 2007.

[34] D. P. Bertsekas and J. N. Tsitsiklis, Parallel and Distributed Computation: Numerical Methods. Belmont, MA: Athena Scientific, 1997.

[35] D. P. Palomar and M. Chiang, "A Tutorial on Decomposition Methods for Network Utility Maximization," IEEE Journal On Selected Areas in Communications, vol. 24, no. 8, pp. 1439-1451, Aug. 2006.

[36] P. Samadi, H. Mohsenian-Rad, R. Schober, V. Wong, and J. Jatskevich, "Optimal real-time pricing algorithm based on utility maximization for smart grids," in Proc of IEEE International Conference on Smart Grid Communications, Gaithersburg, MD, Oct. 2010.

[37] P. Samadi, H. Mohsenian-Rad, R. Schober, and V. Wong, "Advanced demand side management for the future smart grid using mechanism design," IEEE Trans. on Smart Grid, vol. 3, no. 3, pp. 1170-1180, 2012.

[38] D. Dong, F. Lou, X. Zhang, D. Boroyevich, and P. Mattavelli, "Gridinterface bidirectional converter for residential dc distribution systemspart 2: Ac and dc interface design with passive components minimization," IEEE Trans Power Electronics, vol. 28, pp. 1667-1679, Apr. 2013.

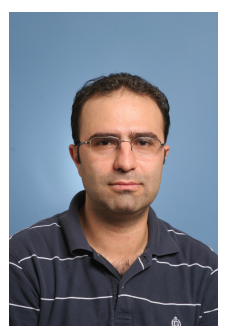

Hamed Mohsenian-Rad (S'04-M'09) received the Ph.D. degree in Electrical and Computer Engineering from the University of British Columbia in Vancouver, Canada in 2008. Currently, he is an Assistant Professor of Electrical Engineering at the University of California at Riverside. Dr. Mohsenian-Rad is the recipient of the NSF CAREER Award, the Best Paper Award from the IEEE International Conference on Smart Grid Communications 2012, and the Best Paper Award from the IEEE Power and Energy Society General Meeting 2013. He serves as Editor for the IEEE Trans. on Smart Grid, the IEEE Communications Surveys and Tutorials, and the IEEE Communications Letters. His research interests are the design, optimization, and game-theoretic analysis of power systems.

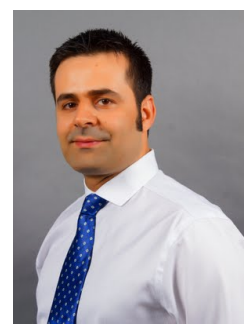

Ali Davoudi (S'04-M'11) received the Ph.D. degree in Electrical and Computer Engineering from the University of Illinois, Urbana, IL, USA, in 2010. He is currently an Assistant Professor at the Electrical Engineering Department of the University of Texas, Arlington, TX, USA. He worked for Solar Bridge Technologies, Texas Instruments Inc., and Royal Philips Electronics. He has served as the editor or guest editor of IEEE Transactions on Power Electronics, Energy Conversion, Smart Grids, Industry Applications, Industrial Informatics, and Vehicular Technology. His research interests are various control aspects of power electronics, energy conversion, and finite-inertia power systems. 